\title{
An Enhanced and Cost Saving Droop Control Method for Improved Load Sharing for the MEA Application
}

\author{
Habibu Hussaini ${ }^{1 *}$, Tao Yang ${ }^{1}$, Cheng Wang ${ }^{1}$, Serhiy Bozhko ${ }^{1}$ \\ ${ }^{1}$ Power Electronics, Machines and Control (PEMC) Research Group, Faculty of Engineering, University of Nottingham, \\ Nottingham, NG7 2RD, UK \\ *Corresponding Author: Habibu.Hussaini@nottingham.ac.uk
}

\begin{abstract}
The more electric aircraft (MEA) concept has been identified as the major trend of future aircraft. The DC distribution network is a promising architecture for moreelectric aircraft application, where multiple electrical sources are connected to a common HVDC bus. The power sharing of these sources is achieved using the droop control method. However, the conventional droop control method has a limitation in achieving accurate load sharing and voltage regulation due to the influence of the cable resistance and nominal voltage reference offset. In this paper, a new method that modifies the droop gain according to the average total cable resistance for improved load sharing is proposed. The overall cable resistance of the system can be estimated based on the measurement of the total load current and knowledge of the value of the DC bus voltage. Also, since the method can be implemented locally without the need for a high-bandwidth communication link, it will save cost and reduce the system complexity. Based on the simulation results obtained, the proposed method has demonstrated better performance when compared to the conventional droop control method for the MEA application for any desired sharing ratio.
\end{abstract}

\section{INTRODUCTION}

The transportation industry is moving towards more electric or all-electric technology due to concerns that stem from environmental pollution and the energy crisis [1]. Some of such example in the transportation industry includes allelectric hybrid vessels [2], hybrid electric vehicles (HEVs) [3] and the more or all-electric aircraft [4]. Many functions which are managed by the secondary power sources (hydraulic, pneumatic and mechanical power) in conventional aircraft can be replaced by electrical power due to the movement towards the more electric aircraft (MEA). This is to improve fuel efficiency, increase the availability and performance of the aircraft, reduce the weight of the aircraft, and reduce the overall cost of maintenance and operation [5]. Consequently, the onboard installed electrical power increases significantly and this results in high electrical power demand. Extracting electric power from both engine shafts thus becomes inevitable. As shown in Fig. 1, the high-pressure shaft and low-pressure shaft within an aircraft engine are each driving a generator and supplying power to a common HVDC bus through an active rectifier.

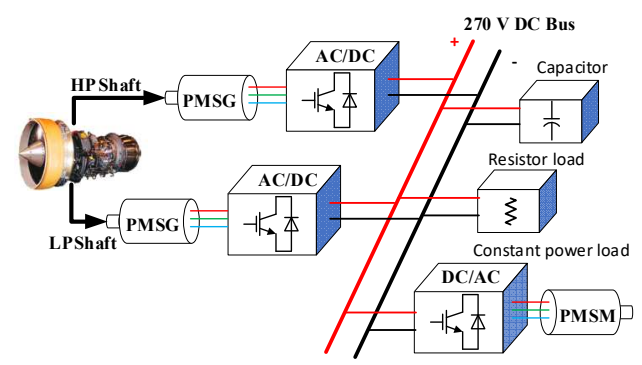

Fig. 1 MEA EPS with HVDC configuration
The choice of the high voltage DC (HVDC) system for the MEA EPS architecture is due to its several advantages when compared to the $\mathrm{AC}$ system. Some of these advantages include higher efficiency, easier control since it does not require synchronization among the generators and no need for reactive power and frequency regulations. This will have a huge impact on the improvement of the system's stability, reliability, power quality and controllability [6]. Also, the parallel-connected converters in the MEA distribution EPS are expected to work together in a coordinated manner to share the load power demand in proportion to their respective power ratings and regulate the DC bus voltage. This is important to ensure that the generators are not overloaded, hence, maintaining the distribution system reliability. The droop control method is usually used for current/load sharing between the sources.

Decentralized control makes use of local measurement to carry out local regulation and this is realized entirely by local controllers and does not require communication between units, hence, improves system reliability, reduce cost and system complexity. Droop control is favourably regarded as a decentralized control method as there is no reliance on a critical communication network. The conventional droop control is achieved by linearly decreasing the DC output voltage as the output current increases [7]. The conventional droop control method has limitation in achieving accurate load sharing and DC bus voltage regulation due to the influence of the cable resistance and nominal voltage reference offset.

Generally, a higher droop gain helps to realize accurate load sharing performance but leads to poor DC bus voltage regulation under heavy load condition. On the other hand, when the droop gain is chosen to be small, the DC bus voltage regulation is enhanced while the load sharing accuracy is degraded [8]. This results in a trade-off between output voltage regulation and accurate load sharing in the low voltage DC microgrid. Therefore, it can be said that the choice of the droop gain plays a vital role in achieving accurate load sharing. Over the years, different methods have been employed to enhance the droop control method to realize accurate load sharing and DC bus voltage regulation. Some of the methods used can achieve accurate load sharing and/or DC bus voltage restoration with the aid of a communication channel [9]. However, this approach will increase the cost of the system and complexity. Other researchers realized improved load sharing by setting droop gain far greater than the cable resistance $\left(k_{d i} \gg R_{i}\right)$ to make the influence of cable resistance on accurate current sharing negligible $[10,11]$ or the cable resistance is completely ignored [12]. However, this is only achievable in a small microgrid where the influence of the cable resistance can be ignored and a relatively high droop gain is set to achieve accurate current/load sharing. In lowvoltage DC microgrid applications such as the more electric 
aircraft, the influence of the cable resistance cannot be ignored $[8,9]$. The line impedance in the low voltage DC microgrid is predominantly resistive [13]. Moreover, a high droop gain will lead to a high DC bus voltage deviation and this will ultimately affect the power quality of the system. Furthermore, the droop gain that can be set is constrained by the maximum allowable voltage deviation of the DC bus voltage and the converter's full load current [8]. Also, some of the methods proposed have practical limitations such as the average loading method proposed in [14] and the line drop compensator method in [8]. In [8], the authors proposed the modification of the effective droop gain according to the corresponding subsystem's cable resistances using a proportional controller. The gain of the proportional controller is set equal to the value of the estimated corresponding subsystem cable resistance. This will help to compensate for the influence of the cable resistance on current sharing. However, the realization of accurate load sharing is entirely dependent on the knowledge (accurate measurement) of the corresponding subsystem cable resistance. The estimation of the line resistance is prone to errors due to the variation of the line resistance with temperature (environmental condition) [15]. Therefore, this approach has a practical limitation. Any error in cable resistance estimation will result in a power sharing error. Moreover, it is difficult in practice to know the value of the corresponding subsystems cable resistance and most of the methods used to estimate the cable resistance require the use of many resources and are based on the injection of disturbances to the system and this has the potential of degrading the power quality of the system and increase the cost.

In this paper, a new control method based on a proportional controller which modifies the droop gain of each subsystem according to the estimated average total cable resistance is proposed to achieve improved load sharing in the more electric aircraft EPS fed by PMSGs. This way the influence of the cable resistance on accurate load sharing is compensated. The method is simple and can be implemented locally, hence, no need for a high-bandwidth communication channel (safe cost and reduce system complexity). Furthermore, it can be implemented practically as only the measurement of the load current is required in the estimation of the total cable resistance.

The rest of the paper is organized as follows. Section II presents the analysis of the conventional droop control method. The proposed average total cable resistance compensation method is discussed in Section III including its principle of operation and method of implementation. The results of the simulation studies carried out to compare the conventional and proposed droop control methods are provided in Section IV. Section V concludes the paper.

\section{ANALYSIS OF LOAD SHARING IN THE CONVENTIONAL DROOP CONTROL METHOD}

The active front end converters connected in parallel and interfaced to the permanent magnet synchronous generators (PMSGs) shown in Fig. 1 can be modelled as an ideal voltage source (with the internal resistance ignored since it is less than the droop resistance) under the droop control strategy. In this paper, we are starting with the basic MEA system with two sources. The multi-source system will be considered in the future. The cable is modelled as resistance for steady-state analysis and the equivalent circuit of the distribution network considering only two sources is as shown in Fig. 2.

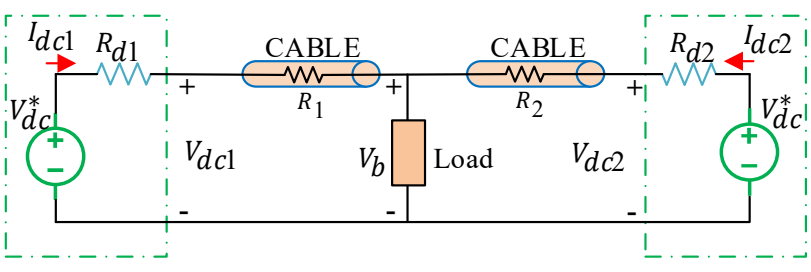

Fig. 2 Steady-state equivalent circuit of the distribution network

The output voltage of the droop controlled converter in Fig. 3 is as expressed in (1).

$$
V_{d c i}=V_{d c i}^{*}-R_{d i} I_{d c i}
$$

where $i=1,2$ represents the converter 1 and 2 respectively connected to the DC microgrid, $V_{d c}{ }^{*}$ represent the nominal voltage reference of the $i$ th DC source under no-load condition, $V_{d c i}$ is the output terminal voltage of the $i$ th DC source, $R_{d i}$ is the equivalent output resistance (or virtual/droop resistance) of the $i$ th DC source, and $I_{d c i}$ is the output current from the $i$ th DC source. Under the no-load condition, $V_{d c l}{ }^{*}=V_{d c 2}{ }^{*}=V_{d c}{ }^{*}$.

The current sharing ratio between the sources in steadystate is as expressed in (2), provided the effect of cable impedance on load sharing is ignored.

$$
I_{d c 1}: I_{d c 2}=\frac{1}{k_{d 1}}: \frac{1}{k_{d 2}}
$$

where $k_{d 1}=R_{d 1}, k_{d 2}=R_{d 2}$ are the coefficients of the droop gain. The droop coefficients are usually selected to be proportional to the generators ratings to ensure an accurate current sharing, based on the assumption that the same nominal voltage $V_{d c}{ }^{*}$ is applied to each of the droop characteristics.

When the voltage drop on the cables is considered and the voltage control dynamics are neglected, the steady-state DC bus voltage as obtained from Fig. 2 is as expressed in (3).

$$
V_{b}=V_{d c i}-R_{i} I_{d c i}=V_{d c}^{*}-\left(R_{d i}+R_{i}\right) I_{d c i}
$$

where $R_{i}$ is the resistance of the cables connecting the $i$ th DC source to the load and $V_{b}$ is the main DC bus voltage. Also, the real power and output current from the individual power converter injected into the system is as expressed in (4) and (5) respectively.

$$
\begin{gathered}
P_{i}=\frac{V_{d c}^{*} V_{b}-V_{b}^{2}}{k_{d i}+R_{i}} \\
I_{d c i}=\frac{V_{d c}^{*}-V_{b}}{R_{d i}+R_{i}}
\end{gathered}
$$

Therefore, from (5), we can obtain the expression in (6)

$$
\frac{I_{d c 1}}{I_{d c 2}}=\frac{k_{d 2}+R_{2}}{k_{d 1}+R_{1}}
$$

Hence, the current sharing between the converters in steady-state is as expressed in (7), assuming the sources are supplying together.

$$
I_{d c 1}: I_{d c 2}=\frac{1}{k_{d 1}+R_{1}}: \frac{1}{k_{d 2}+R_{2}}
$$


where $k_{d 1}=R_{d 1}, k_{d 2}=R_{d 2}$ are the droop coefficients of the individual converter connected in parallel.

It can be observed from (7) that the power sharing ratio of the sources will be impacted by both the cable resistance and droop gain. Hence, power sharing accuracy among the sources may not be as desired using the conventional droop control method. The inaccurate power sharing can be attributed to the voltage deviation in the droop controller and the difference in the output impedance of the power converters as a result of the difference in cable impedance.

The maximum droop gain that can be set is constrained by the maximum allowable voltage deviation of the DC bus voltage and the converter's full load current as expressed in (8) [8].

$$
k_{d \max }=\frac{\Delta V_{\text {bmax }}}{i_{F}}
$$

where the power converter's full load current is represented as $i_{F}, k_{d \max }$ is the maximum allowable droop gain and $\Delta V_{b \max }$ is the maximum allowable deviation of the DC bus voltage.

Also, when the influence of cable resistance is considered, this modifies the effective droop gain. This causes the effective droop gain to increase from $k_{d i}$ in (2) to $k_{d i}+R_{i}$ in (7), hence, leading to poor regulation of the DC bus voltage. On the other hand, accurate load sharing is not realized due to the unequal cable resistances connecting the parallel-connected DC sources to the load. The unequal cable impedance which is usually a common feature of a low voltage distribution system can be attributed to the difference in the relative distance (geographic location) between the DC sources and the load in the microgrid [8].

However, the MEA EPS distribution network in which this proposed method is desired to be applied, the generators are located at approximately the same distance (cable length) from the power distribution centre. In order words, the cable resistance from each of the generators to the load can be assumed to be identical due to the symmetrical geometry of the MEA electrical power system [15]. The problem here is that the conventional droop control method can only realize accurate current sharing (since the cable resistance is assumed to be the same) when the desired sharing ratio is equal. Therefore, there is a need to find a way of ensuring improved/accurate load/power sharing between the parallelconnected sources in the MEA EPS using the droop control method (since the method is independent of a communication link between the parallel-connected source converters) in steady-state for any desired sharing ratio.

\section{Proposed Average Total Cable Resistance COMPENSATION CONTROL METHOD}

\section{A. Principle of Operation and Implementation of Proposed Droop Control Method}

This method involves the modification of the corresponding subsystems droop gain according to the average of the total cable resistance. The droop gain modification is realized through the addition of a compensation term to each of the subsystem (locally) using a proportional controller as shown in Fig. 3. This control method works similarly as a virtual negative resistance to mitigate the effect of the cable resistance on accurate current sharing through compensation.

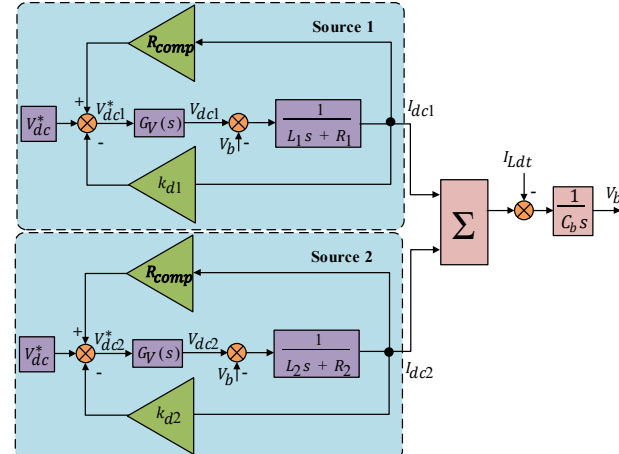

Fig. 3 Proposed method control scheme in the voltage-mode droop control method

The control dynamics of the $i$ th converter is denoted as $\mathrm{Gv}(\mathrm{s})$ in Fig. 3. A common compensation term $\left(R_{c o m p} I_{d c i}\right)$ is added to the terminal voltage reference for each of the subsystem (module) as shown in Fig. 3. The feedback variable of the proportional controller is the output DC current $\left(I_{d c i}\right)$ and the value of the estimated average total cable resistance $\left(R_{\text {comp }}\right)$ is set as the gain of the proportional controller. Therefore, load sharing error caused by the conventional droop control method due to the influence of the corresponding subsystems cable resistance is compensated and improved load sharing can be realized for any desired sharing ratio.

In the proposed control method, there is no need for highbandwidth communication between the power converters as they can operate independently, hence, will reduce system cost and complexity. Furthermore, the proposed method is simple and can be implemented locally. The method is easy to implement and practicable as the only measurement that is required is that of the total load current and no need for any voltage controllers.

\section{A. Mathematical Analysis of the Proposed Method}

When the compensation term is added, and the voltage control dynamics are neglected, the new steady-state DC bus voltage is expressed as

$$
V_{\text {bnew }}=V_{d c}^{*}-\left(k_{d i}+R_{i}\right) I_{d c i}+R_{c o m p} I_{d c i}
$$

Hence, from (9), the new or modified droop gain due to the compensation term and the new current sharing ratio for the proposed droop control method is as expressed in (10) and (11) respectively.

$$
\begin{gathered}
k_{\text {dinew }}=k_{d i}-R_{\text {comp }} \\
I_{\text {dc1 new }}: I_{d c 2 \text { new }}=\frac{1}{k_{\text {d1new }}+R_{1}}: \frac{1}{k_{d 2 n e w}+R_{2}} \\
R_{\text {comp }}=\frac{\sum_{i=1}^{2} R_{i}}{2}
\end{gathered}
$$

where $R_{\text {comp }}$ is the average total cable resistance, $\sum_{i=1}^{2} R_{i}$ is the estimated total cable resistance and $k_{\text {dinew }}$ is the modified (new) droop gain due to the introduction of the compensation term. Fig. 4 shows the control block diagram of the implementation of the proposed enhanced droop control method (for the voltage-mode droop control scheme) for the MEA single bus DC microgrid distribution network fed by a 
PMSG working in flux weakening and generation mode. Also, the proposed control method is implemented locally as shown in Fig. 4.

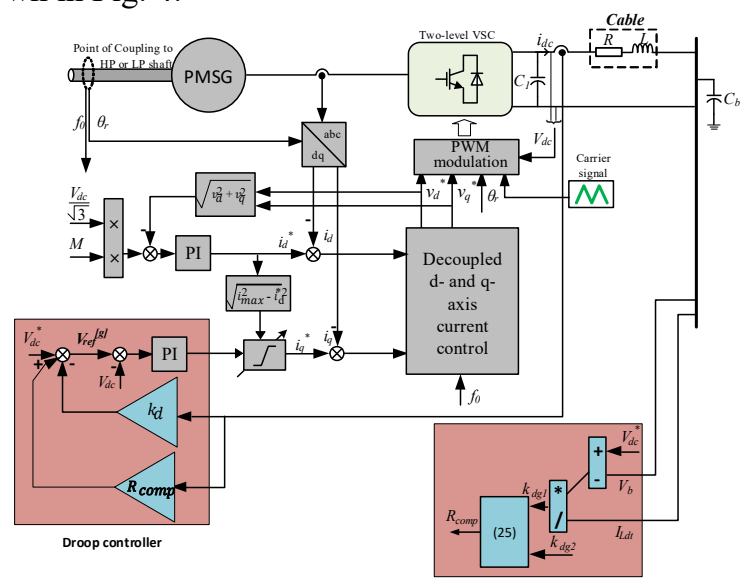

Fig. 4 Control diagram of the proposed droop control method implemented for the MEA application

\section{B. Estimation of the Total Cable Resistance}

It is a well-known fact that in a DC system controlled using the droop control method, the main DC bus voltage decreases as the load power or current increases. In order words, the DC bus voltage will decrease under heavy load conditions due to an increase in the load power or current. To compensate for the voltage deviation associated with the main DC bus, the idea of the global droop gain was proposed in $[15,16]$. Just as the individual subsystem in a multi-source system controlled by the droop control method have their droop gain, the global droop coefficient helps to define the relationship (V-I characteristics) between the main DC bus voltage and the total load current. Therefore, in this paper, the concept of the global droop gain is used in the estimation of the total cable resistance as follows.

The DC bus voltage for the conventional droop control method is expressed in (3). Therefore, for a two-source voltage droop controlled system, the DC bus voltage is expressed in (13) [16].

$$
V_{b}=V_{d c}^{*}-\left(k_{d 1}+R_{1}\right) I_{d c 1}=V_{d c}^{*}-\left(k_{d 2}+R_{2}\right) I_{d c 2}
$$

where the branch currents and their respective subsystem droop gains are represented as $I_{d c 1}, \quad I_{d c 2}$ and $k_{d l}, k_{d 2}$ respectively; the main DC bus nominal voltage is $V_{d c}{ }^{*}$ (270 $\mathrm{V})$, and the main DC bus voltage is $\mathrm{V}_{\mathrm{b}}, R_{1}$ and $R_{2}$ are the resistance of the cables. Hence, the sum of the subsystem branch currents that make up the total load current can be expressed as in (14).

$$
I_{L d t}=I_{d c 1}+I_{d c 2}=\left(V_{d c}^{*}-V_{b}\right) \sum_{i=1}^{2} \frac{1}{k_{d i}+R_{i}}
$$

where $I_{L d t}$ is the total load current, which can be measured on the bus bar of the EPS as shown in Fig. 4. The bus bar (main feeder) supplies power to all the loads connected to the system. The expression in (14) can be rewritten as shown in (15) to show the V-I relationship between the main DC bus voltage and the total load current.

$$
V_{b}=V_{d c}^{*}-I_{L d t} \frac{1}{\sum_{i=1}^{2} \frac{1}{k_{d i}+R_{i}}}
$$

Furthermore, the expression in (15) can be re-written as in (16) and (17).

$$
\begin{gathered}
V_{b}=V_{d c}^{*}-I_{L d t} k_{d g 1} \\
k_{d g 1}=\frac{V_{d c}^{*}-V_{b}}{I_{L d t}}
\end{gathered}
$$

where $k_{d g l}$ is the global droop gain. Again, from (15), the global droop gain can also be expressed as in (18)

$$
k_{d g 1}=\frac{1}{\sum_{i=1}^{2} \frac{1}{k_{d i}+R_{i}}}
$$

where the cable resistance is represented by $R_{i}$. It can be observed that the global droop gain $\left(k_{d g l}\right)$ cannot be calculated directly from (18), this is because the corresponding subsystems resistance $\left(R_{l}\right.$ and $\left.R_{2}\right)$ are not known. Hence, $\left(k_{d g l}\right)$ will be determined from (17). Therefore, in this paper, $k_{d g l}$ can be obtained by the measurement of the total load current $\left(I_{L d t}\right)$ and DC bus voltage $\left(V_{b}\right)$ as expressed in (17) and shown in Fig. 4. Furthermore, the expression of the global droop gain for the conventional droop control method in an ideal situation whereby the effect of the cable resistance is negligible is as expressed in (19).

$$
k_{d g 2}=\frac{1}{\sum_{i=1}^{2} \frac{1}{k_{d i}}}
$$

It can be observed from (19), that the value of $k_{d 2}$ depends on the converters droop coefficients. Since $k_{d 1}$ and $k_{d 2}$ are assigned by the controller, hence, the value of $k_{d g 2}$ can be obtained from the controller.

Now, based on the expressions of global droop gains in (18) and (19) we can develop an expression for the estimation of the total cable resistance for a multi-source droop controlled system. However, in this paper, since we are only considering two sources for the ease of analysis, the total cable resistance estimation analysis is as follows.

From (18) and (19), the expressions for $k_{d g l}$ and $k_{d g 2}$ can be re-written as in (20) and (21) respectively, for a two-source system (in this case, $n=2$ ).

$$
\begin{gathered}
k_{d g 1}=\frac{1}{\frac{1}{k_{d 1}+R_{1}}+\frac{1}{k_{d 2}+R_{2}}}=\frac{k_{d 1} k_{d 2}+R_{1} R_{2}+k_{d 1} R_{2}+k_{d 2} R_{1}}{k_{d 1}+k_{d 2}+R_{1}+R_{2}} \\
k_{d g 2}=\frac{1}{\frac{1}{k_{d 1}}+\frac{1}{k_{d 2}}}=\frac{k_{d 1} k_{d 2}}{k_{d 1}+k_{d 2}}
\end{gathered}
$$

By dividing the numerator and denominator of the expression in (20) by $k_{d 1} k_{d 2}$, we obtain the expression in (22).

$k_{d g 1}=\frac{\frac{k_{d 1} k_{d 2}}{k_{d 1} k_{d 2}}+\frac{R_{1} R_{2}}{k_{d 1} k_{d 2}}+\frac{k_{d 1} R_{2}}{k_{d 1} k_{d 2}}+\frac{k_{d 2} R_{1}}{k_{d 1} k_{d 2}}}{\frac{k_{d 1}+k_{d 2}}{k_{d 1} k_{d 2}}+\frac{R_{1}+R_{2}}{k_{d 1} k_{d 2}}}=\frac{1+\frac{R_{1} R_{2}}{k_{d 1} k_{d 2}}+\frac{R_{2}}{k_{d 2}}+\frac{R_{1}}{k_{d 1}}}{\frac{1}{k_{d g 2}}+\frac{R_{1}+R_{2}}{k_{d 1} k_{d 2}}}$

Also, assuming $k_{d 1} \gg R_{1}$ and $k_{d 2} \gg R_{2}$, for this analysis, we obtain

$$
\frac{1+0+0+0}{\frac{1}{k_{d g 2}}+\frac{R_{1}+R_{2}}{k_{d 1} k_{d 2}}} \approx k_{d g 1}
$$




$$
R_{1}+R_{2} \approx\left(\frac{1}{k_{d g 1}}-\frac{1}{k_{d g 2}}\right) k_{d 1} k_{d 2}
$$

Hence, the total cable resistance can be approximated to the expression in (24) because of the assumption made earlier to ease the mathematical analysis. Now, the average total cable resistance $\left(R_{\text {comp }}\right)$ expressed in (12) can be re-written as in $(25)$.

$$
R_{\text {comp }}=\frac{R_{1}+R_{2}}{2} \approx \frac{\left(\frac{1}{k_{d g 1}}-\frac{1}{k_{d g 2}}\right) k_{d 1} k_{d 2}}{2}
$$

\section{Estimation of Error in Current Sharing Performance}

In order to compare the current sharing performance between the conventional droop control method and the proposed method for different desired sharing ratio, the mathematical expression in (26) is used. The expression in (26) is used to calculate the percentage error in the current sharing ratio based on the desired sharing ratio and the actual sharing ratio realized by each of the methods. The expression is only applicable for a system containing only two sources as is the case in this paper.

$$
\operatorname{Error}(\%)=\left|\frac{n_{\text {desired }}-n_{\text {actual }}}{n_{\text {desired }}}\right| \times 100 \%
$$

where $n_{\text {desired }}$ is the desired sharing ratio and $n_{\text {actual }}$ is the actual sharing ratio obtained by using the conventional and proposed droop control method.

\section{Simulation STUdieS}

Simulations were carried out to compare the accuracy of the load sharing performance between the conventional and proposed droop control method. The topology used for the simulation is as shown in Fig. 5 and can be regarded as a basic architecture of the more electric aircraft EPS. Moreover, the MEA EPS make use of two main generators and the auxiliary power unit (APU) is only used together with one of the generators during an emergency. This topology is made up of two twin converters $\left(\mathrm{AR}_{1-2}\right)$ operating in parallel and a constant power load. However, the proposed method can be used in a system with more than two sources.

The system is modelled using MATLAB SIMULINK C for both the conventional and proposed droop control method. The CPL of $20 \mathrm{~kW}$ is applied to the system at $0.2 \mathrm{~s}$ and increased by steps of $+10 \mathrm{~kW}$ at $0.25 \mathrm{~s}$, and $0.27 \mathrm{~s}$ during the simulation. The simulation was run for $0.3 \mathrm{~s}$. The system parameters used for the simulations are as shown in TABLE I. The simulation is carried out with equal cable resistance (i.e equal cable lengths) since the cables connecting the generators to the load in the MEA can be assumed to be of equal lengths. The desired load sharing ratio is $1: 2$ based on the droop gain settings in TABLE I. The equivalent DC cable parameters used in the simulations are as shown in TALE II.

The simulation results obtained for the current sharing between the two generators using the conventional and proposed droop control method are as summarized in TABLE III. Fig. 6 (a) and (b) also show the simulation results for the output DC currents and DC bus voltage respectively in graphical form for the desired sharing ratio of 1:2.

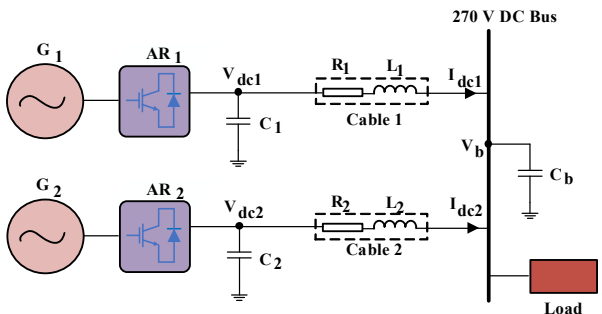

Fig. 5: Topology used in the simulation

TABLE I: ElECTRICAL POWER SYSTEM (EPS) PARAMETERS

\begin{tabular}{lcl}
\hline \hline Parameter & Symbol & Value \\
\hline \hline Rated Voltage of main DC Bus & $V_{d c}{ }^{*}$ & $270 \mathrm{~V}$ \\
Local Shunt Capacitor & $C_{i}$ & $1.2 \mathrm{mF}$ \\
Main DC bus capacitor & $C_{b}$ & $0.6 \mathrm{mF}$ \\
Converter 1 Droop gain & $k_{d 1}$ & $1 / 4.250$ \\
Converter 2 Droop gain & $k_{d 2}$ & $1 / 8.50$ \\
\hline \hline
\end{tabular}

TABLE II: EQUIVALENT DC CABLES PARAMETERS Resistance $\left(R_{i}\right)-\quad$ Inductance $\left(L_{i}\right)-\quad$ Length $(\mathrm{m})$ $(0.6 \mathrm{~m} \Omega / \mathrm{m}) \quad(0.2 \mu \mathrm{H} / \mathrm{m})$

\begin{tabular}{llll} 
& $(0.6 \mathrm{~m} \Omega / \mathrm{m})$ & $(0.2 \mu \mathrm{H} / \mathrm{m})$ & \\
\cline { 2 - 4 } Cable 1 & $30 \mathrm{~m} \Omega$ & $10 \mu \mathrm{H}$ & 50 \\
\hline Cable 2 & $30 \mathrm{~m} \Omega$ & $10 \mu \mathrm{H}$ & 50 \\
\hline \hline
\end{tabular}

TABLE III: SUMMARY OF SIMULATION RESULTS AT T $=0.27 \mathrm{~S}$

\begin{tabular}{l|cccccc}
\hline \hline $\begin{array}{l}\text { Conventional } \\
\text { Method }\end{array}$ & $k_{d 1}$ & $k_{d 2}$ & $I_{d c 1}$ & $I_{d c 2}$ & Ratio & $\begin{array}{c}\text { Error } \\
(\%)\end{array}$ \\
\cline { 2 - 7 } & $1 / 4.250$ & $1 / 8.50$ & 56.06 & 100.70 & $1: 1.8$ & 10 \\
\hline $\begin{array}{l}\text { Proposed } \\
\text { Method }\end{array}$ & $k_{d 1 \text { new }}$ & $k_{d 2 \text { new }}$ & $I_{d c I n e w}$ & $I_{d c 2 \text { new }}$ & Ratio & $\begin{array}{c}\text { Error } \\
(\%)\end{array}$ \\
\cline { 2 - 7 } & $1 / 4.871$ & $1 / 11.41$ & 51.70 & 103.40 & $1: 2$ & 0
\end{tabular}

It is observed that the current sharing ratio among the two converters in steady-state using the conventional droop control methods is $1: 1.8$ as shown in TABLE III. This clearly shows that the result obtained is not in the desired sharing ratio of $1: 2$ and the percentage error in the current sharing ratio is calculated to be $10 \%$. The inaccurate current sharing in the conventional droop control methods is due to the influence of the cable resistance. On the other hand, the proposed droop control method can achieve the desired sharing ratio of $1: 2$ as shown in TABLE III. This shows that the average total cable resistance can effectively compensate for the effect of the corresponding subsystem cable resistance on current sharing.

Furthermore, in both the conventional and proposed droop control methods, the DC bus voltage decreases as the load current increases. When a constant power load of $40 \mathrm{~kW}$ is applied to the system at $0.27 \mathrm{~s}$, the main DC bus voltage dropped to $255.1 \mathrm{~V}\left(V_{b c o n v}\right)$ and $257.8 \mathrm{~V}\left(V_{\text {bprop }}\right)$ from its initial value of $270 \mathrm{~V}$ due to the increase in the load current for both the conventional and proposed droop control methods respectively as shown in Fig. 6b. However, the proposed method can slightly improve the DC bus voltage regulation due to the compensation of the cable impedance.

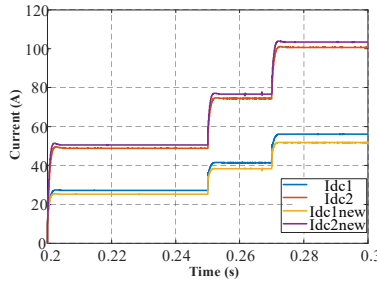

(a)

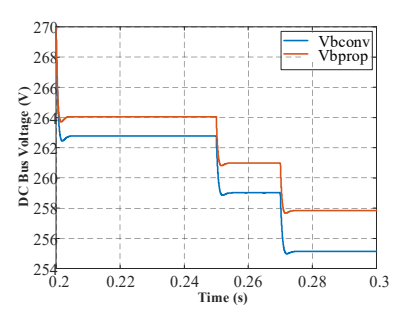

(b) 
Fig. 6: Simulation Results for Comparing the Performance of the Conventional and the Proposed Droop Control Methods for the Desired Current Sharing Ratio (1:2) (a) DC Currents (b) Bus Voltage

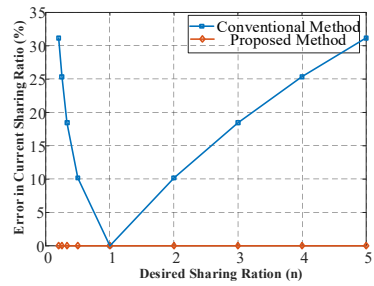

Fig. 7: Comparison between conventional and proposed droop control methods under different current sharing ratios

To further compare the current sharing performance between the conventional and proposed droop control methods, different sharing ratios are used. Desired sharing ratios of $1: 5,1: 4,1: 3,1: 2,1: 1,2: 1,3: 1,4: 1$ and $5: 1$ are used and are represented with their decimal equivalents in the horizontal axis of Fig. 7. For each of the desired sharing ratio, the output DC currents from the converters are obtained from simulations and the corresponding percentage error in the current sharing ratio is calculated and the results obtained are as shown in Fig. 7 for both the conventional and proposed droop control methods.

It is observed from Fig. 7 that the proposed method can realize accurate current sharing without error under the various load sharing ratios desired. On the other hand, the conventional droop control method performs poorly in almost all the desired sharing ratio when compared to the proposed method. The worst performance is observed at desired sharing ratios of 1:5 and 5:1 with a percentage error in the current sharing ratio of $31 \%$ in both cases. Also, the percentage error in the current sharing ratio of the conventional droop control method increases with an increase in sharing ratio between the generators. However, both the conventional and proposed droop control method have similar current sharing performance (with $0 \%$ error in the current sharing ratio) when the desired sharing ratio is $1: 1$ as expected due to the equal cable lengths from each of the generators to the load.

\section{CONCLUSION}

In this paper, a new enhanced droop control method has been proposed for the MEA application. The proposed method can be effectively applied in a system such as the more electric aircraft whose cable lengths can be assumed to be equal (from each generator to the load) for accurate load sharing. The proposed control method is simple, can be implemented locally without the need for a high-bandwidth communication link, hence, will save cost and reduce system complexity.

In future, we intend to use artificial intelligence to develop an artificial neural network (ANN) model based on sample data obtained from simulation studies to select the optimal droop coefficient for the droop control method employed in DC microgrids irrespective of the variation in the corresponding subsystems cable resistance. Also, we intend to propose a method for the DC bus restoration based on the proposed modified droop gain for the MEA application.

\section{ACKNOWLEDGMENT}

This project has received funding from the Clean Sky 2 Joint Undertaking under the European Union's Horizon 2020 research and innovation programme under grant agreement No 807081. The author Habibu Hussaini extends his profound appreciation to the Petroleum Technology Development Fund (PTDF), Nigeria, for the scholarship funding.

\section{REFERENCES}

[1] J. Chen, C. Wang and J. Chen, "Investigation on the selection of electric power system architecture for future more electric aircraft," IEEE Transactions on Transportation Electrification, vol. 4, no. 2, pp. 563-576, 2018.

[2] L. W. Chua, T. Tjahjowidodo, G. G. Seet and R. Chan, "Implementation of optimization-based power management for allelectric hybrid vessels," IEEE Access, vol. 6, pp. 74339-74354, 2018.

[3] A. Emadi, K. Rajashekara, S. S. Williamson and S. M. Lukic, "Topological overview of hybrid electric and fuel cell vehicular power system architectures and configurations," IEEE Transactions on vehicular technology, vol. 54, no. 3, pp. 763-770, 2005.

[4] P. Wheeler, "Technology for the more and all electric aircraft of the future," in 2016 IEEE International Conference on Automatica (ICA$A C C A$ ), October 2016, pp. 1-5.

[5] J. A. Rosero, J. A. Ortega, E. Aldabas and L. A. R. L. Romeral, "Moving towards a more electric aircraft," IEEE Aerospace and Electronic Systems Magazine, vol. 22, no. 3, pp. 3-9, 2007.

[6] D. Kumar, F. Zare and A. Ghosh, "DC microgrid technology: system architectures, AC grid interfaces, grounding schemes, power quality, communication networks, applications, and standardizations aspects," IEEE Access, vol. 5, pp. 12230-12256, 2017.

[7] X. Lu, J. M. Guerrero, K. Sun and J. C. Vasquez, " An improved droop control method for dc microgrids based on low bandwidth communication with dc bus voltage restoration and enhanced current sharing accuracy," IEEE Transactions on Power Electronic, vol. 29, no. 4, pp. 1800-1812, April 2014

[8] A. Tah and D. Das, " An enhanced droop control method for accurate load sharing and voltage improvement of isolated and interconnected DC microgrids," IEEE Transactions on Sustainable Energy, vol. 7, no. 3, pp. 1194-1204, 2016.

[9] N. Yang, D. Paire, F. Gao, A. Miraoui and W. Liu, "Compensation of droop control using common load condition in DC microgrids to improve voltage regulation and load sharing," International Journal of Electrical Power \& Energy Systems, vol. 64, pp. 752-760, 2015.

[10] T. Dragičević, J. M. Guerrero, J. C. Vasquez and D. Škrlec, "Supervisory control of an adaptive-droop regulated DC microgrid with battery management capability," IEEE Transactions on power Electronics, vol. 29, no. 2, pp. 695-706, Feb. 2014

[11] J. Chen and Q. Song, "A decentralized dynamic load power allocation strategy for fuel cell/supercapacitor-based APU of large more electric vehicles," IEEE Transactions on Industrial Electronics, vol. 66, no. 2, pp. 865-875, Feb. 2019.

[12] K. Sun, L. Zhang, Y. Xing and J. M. Guerrero, "A distributed control strategy based on DC bus signaling for modular photovoltaic generation systems with battery energy storage," IEEE Transactions on Power Electronics, vol. 26, no. 10, pp. 3032-3045, 2011.

[13] Z. Shuai, D. He, J. Fang, Z. J. Shen, C. Tu and J. Wang, "Robust droop control of DC distribution networks," IET Renewable Power Generation, vol. 10, no. 6, pp. 807-814, 2016.

[14] S. Anand, B. G. Fernandes and J. Guerrero, "Distributed control to ensure proportional load sharing and improve voltage regulation in low-voltage DC microgrids," IEEE Transactions on Power Electronics, vol. 28, no. 4, pp. 1900-1913, April 2013.

[15] F. Gao, S. Bozhko, G. Asher, P. Wheeler and C. Patel, "An Improved Voltage Compensation Approach in a Droop-Controlled DC Power System for the More Electric Aircraft," IEEE Transactions on Power Electronics, vol. 31, no. 10, pp. 7369 - 7383, 2016.

[16] F. Gao, S. Bozhko and P. Wheeler, "An enhanced secondary control approach for voltage restoration in the DC distribution system," SAE Technical Paper, No. 2016-01-1985, 2016. 